ARTICLE

\title{
A randomized trial evaluating virus-specific effects of a combination probiotic in children with acute gastroenteritis
}

Stephen B. Freedman (10 ${ }^{1 凶}$, Jianling Xie ${ }^{1}$, Alberto Nettel-Aguirre ${ }^{1}$, Xiao-Li Pang $^{2}$, Linda Chui ${ }^{2}$, Sarah Williamson-Urquhart', David Schnadower ${ }^{3}$, Suzanne Schuh ${ }^{4}$, Philip M. Sherman (D ${ }^{4}$, Bonita E. Lee ${ }^{2}$, Serge Gouin ${ }^{5}$, Ken J. Farion (10 ${ }^{6}$, Naveen Poonai ${ }^{7}$, Katrina F. Hurley ${ }^{8}$, Yuanyuan Qiu ${ }^{2}$, Binal Ghandi ${ }^{2}$, Colin Lloyd ${ }^{2} \&$ Yaron Finkelstein ${ }^{4}$, the Pediatric Emergency Research Canada Probiotic (PERC) Regimen for Outpatient Gastroenteritis Utility of Treatment (PROGUT) Trial Group*

Gastroenteritis accounts for nearly 500,000 deaths in children younger than 5 years annually. Although probiotics have been touted as having the potential to expedite diarrhea resolution, recent clinical trials question their effectiveness. A potential explanation is a shift in pathogens following the introduction of a rotavirus vaccine. Here, we report the results of a multi-center, double-blind trial of 816 children with acute gastroenteritis who completed follow-up and provided multiple stool specimens. Participants were randomized to receive a probiotic containing Lactobacillus rhamnosus and Lactobacillus helveticus or placebo. We report no virus-specific beneficial effects attributable to the probiotic, either in reducing clinical symptoms or viral nucleic acid clearance from stool specimens collected up to 28 days following enrollment. We provide pathophysiological and microbiologic evidence to support the clinical findings and conclude that our data do not support routine probiotic administration to children with acute gastroenteritis, regardless of the infecting virus.

\footnotetext{
${ }^{1}$ Alberta Children's Hospital Foundation Professor in Child Health and Wellness, Alberta Children's Hospital Research Institute, Cumming School of Medicine, University of Calgary, 28 Oki Drive NW, Calgary, AB T3B 6A8, Canada. ${ }^{2}$ University of Alberta, 116 St \& 85 Ave., Edmonton, AB T6G 2R3, Canada. ${ }^{3}$ University of Cincinnati, 3333 Burnet Ave, Cincinnati, OH UCA 45229, USA. ${ }^{4}$ University of Toronto, 555 University Avenue, Toronto, ON M5G 1X8, Canada. ${ }^{5}$ Université de Montréal, 3175 Chemin de la Côte-Sainte-Catherine, Montréal, QC H3T 1C5, Canada. ${ }^{6}$ University of Ottawa, 401 Smyth Rd, Ottawa, ON K1H 8L1, Canada. 7 University of Western Ontario, 800 Commissioners Road E, London, ON N6A 5W9, Canada. ${ }^{8}$ Dalhousie University, 5980 University Avenue, PO Box 9700 Halifax, NS B3K 6R8, Canada. ${ }^{\star} A$ list of authors and their affiliations appears at the end of the paper. ${ }^{凶}$ email: Stephen.freedman@ahs.ca
} 
$\mathrm{t}$ is estimated that 0.57 acute gastroenteritis (AGE) episodes per person-year occur in Canada, amounting to nearly 19.5 million episodes annually ${ }^{1}$, while in the United States, over 48 million episodes occur each year ${ }^{2,3}$. Despite the availability of a vaccine against rotavirus ${ }^{4}$, which has led to precipitous decreases in hospitalizations ${ }^{5}$ and emergency department (ED) visits ${ }^{6}$ attributable to rotavirus gastroenteritis, coverage remains far from universal; on the global scale, rotavirus remains the leading etiology of diarrhea-associated mortality ${ }^{7}$. Other viruses also contribute significantly to the burden of disease in North America, where norovirus now represents the leading etiology of medically-attended $\mathrm{AGE}^{8,9}$. Because there are no widely accepted effective treatment options available beyond supportive care, health care providers and affected individuals continue to explore a variety of options, including probiotics ${ }^{10}$.

Although the administration of probiotic agents to children with AGE and diarrhea has been recommended by international clinical guidelines ${ }^{11-13}$, we recently conducted one of the largest randomized clinical trials to date, and found no benefits associated with probiotic administration. The trial, which included 886 children with AGE ( 816 of whom completed provided a stool specimen and completed follow-up), reported that those who received a 5-day course of $L$. helveticus/L. rhamnosus did not have significantly different odds of experiencing moderate-to-severe AGE following randomization compared with those administered a placebo [OR: $1.06(95 \% \mathrm{CI}, 0.77$ to 1.46$)]^{14}$. These findings were supported by a simultaneously conducted trial in the United States, which evaluated a different probiotic product, containing L. rhamnosus $\mathrm{GG}^{15}$. A potential explanation for the lack of benefit associated with probiotic administration in these studies is that the benefits may be pathogen specific (e.g., beneficial in rotavirus infection but not norovirus $)^{16}$. This explanation is supported by the diverse underlying pathophysiologic processes induced by different etiologic pathogens ${ }^{17}$ and the multiple proposed mechanisms of action of probiotics ${ }^{18}$.

Understanding pathogen-specific effects is increasingly important since rotavirus vaccination programs have substantially altered the target pathogen population. Advances in molecular diagnostics have also enabled the identification of enteropathogens in more than $75 \%$ of stool specimens submitted by children with $\mathrm{AGE}^{19}$, revealing a shift from rotavirus to norovirus as the most common identified pathogen among individuals with AGE seeking medical care in the United States ${ }^{8,20}$. Moreover, real-time reverse transcription-polymerase chain reaction testing has identified a wide range of viral loads in norovirus-associated AGE cases $^{21}$ with high loads being associated with more severe symptoms ${ }^{22-24}$, prolonged hospitalization and viremia ${ }^{23,25}$. After attaining a peak level during an AGE episode, stool viral loads decrease in a time-dependent manner; the higher the initial viral load, the longer the time required for clearance from stool ${ }^{26}$. As such, the ability to reduce the intestinal viral load more rapidly would represent an objective, pathogenspecific method of how probiotics could modulate AGE infections.

The aforementioned shift in etiologic pathogens, the ability to identify pathogens in real-time ${ }^{27}$, the huge market share and concerns regarding money spent on probiotics ${ }^{28}$ and the recent publication of two studies that question the benefits commonly touted for probiotics ${ }^{14,15}$, highlight the importance of understanding the pathophysiologic pathogen-specific potential benefits of probiotic administration. Because the aforementioned clinical trials relied on caregiver report of clinical symptoms, they did not explore potential therapeutic effects at individual patient and pathogen levels.

To address this point, as an integral part of the aforementioned placebo-controlled, randomized, parallel-arm, clinical trial ${ }^{14}$, we identify pathogens in collected stool specimens to enable an evaluation of the ability of an orally administered probiotic to reduce symptom severity at a pathogen-specific level. We also assess the changes, from baseline, in viral loads in stool specimens at the end of the probiotic course (on day 5 of treatment) and 4 weeks after randomization (on day 28), relative to placebo. We determine that the probiotic has no pathogen-specific beneficial effects compared to placebo, either in reducing clinical symptoms or clearance of viral nucleic acid from stool specimens collected up to 28 days following enrollment. We provide pathophysiological and microbiologic evidence to support the clinical findings, and conclude that our data do not support routine probiotic administration to children with AGE.

\section{Results}

Participants. Of the 886 children who were enrolled into the clinical trial between November 5, 2013, and April 7, 2017, 816 (92.1\%) provided a baseline stool specimen and completed follow-up; Fig. 1. Demographic and clinical characteristics of the probiotic and placebo groups are summarized in Table 1; there were no meaningful differences in index visit clinical parameters. A virus was only detected in 451 (55.3\%) children, bacteria only in $37(4.5 \%)$, virus/bacteria co-detection in $19(2.3 \%)$, parasite-viral/parasite co-detection in $10(1.2 \%)$, and no pathogen was identified in 299 (36.6\%) participants; Supplementary Table 2. There were differences noted regarding the distribution of pathogens among patient groups with respect to age, rotavirus vaccination status, presence of vomiting, and number of diarrhea episodes; supplementary Table 3.

Primary outcome. No differences were detected in the mean post-randomization modified Vesikari scale (MVS) scores between probiotic and placebo groups for any of the five categories of pathogens analyzed; Table 2 and Fig. 2. The weighted linear regression model fitted with interaction terms and covariates revealed no significant associations between postrandomization MVS scores and treatment allocation; supplementary Table 4 . The interaction terms were removed from the regression model as they were not significant; after removal, the findings were unchanged; supplementary Table 5. Statistically significant associations with the post-randomization MVS score were identified for age $(-0.054$; 95\%CI: $-0.083,-0.025$ per 1 month increase) and baseline MVS score (0.339; 95\%CI: 0.198, 0.480 per 1 point increase). In our linear regression models that included a priori identified covariates and only individual pathogen groups and pathogens, no significant association between the post-randomization MVS score and treatment allocation were identified for the following groups: test negative, isolated bacteria, isolated virus, virus/bacteria co-detection, parasite and parasite/virus co-detection, adenovirus, norovirus, rotavirus, and Campylobacter spp.; Supplementary Tables 6-17.

Secondary outcomes. There were no clinically significant differences between the 148 children who provided all three stool samples and those who provided only one or two samples; Supplementary Table 18. An insufficient number of participants had bacterial infections to enable a statistically robust analysis of bacterial pathogen load reduction. Comparing the pathogen load reductions among participants with adenovirus, norovirus, or rotavirus, there were no significant differences between participants administered probiotic or placebo between days 0 to 5 , and 5 to 28; Table 3. Probiotic administration was not associated with pathogen load reduction in any of the 9 linear regression models constructed for the 3 viruses (i.e., adenovirus, norovirus, rotavirus) and 2 time intervals (i.e., 0 to 5 or 5 to 28 days). 


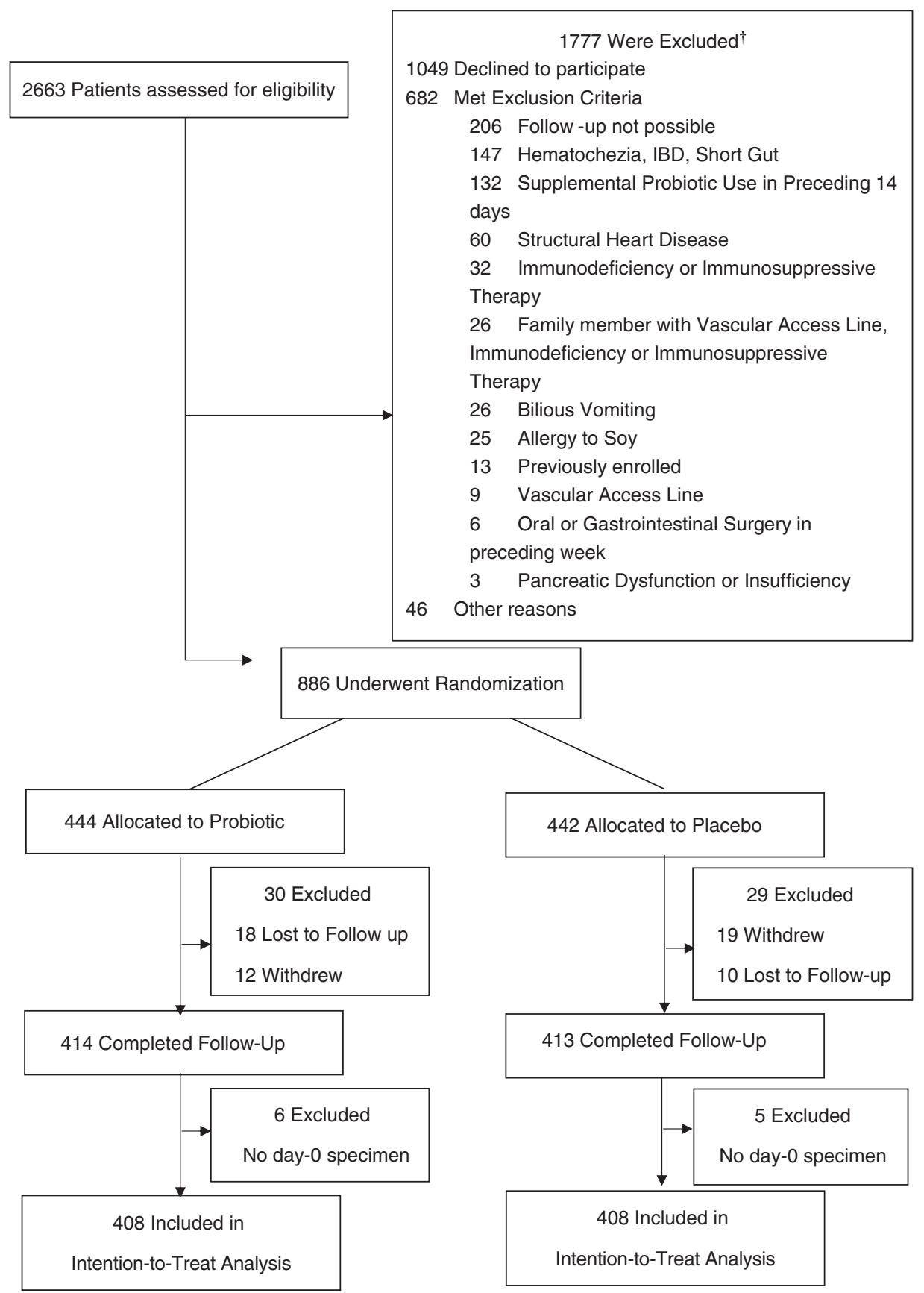

Fig. 1 Flow diagram of patient cohort. IBD inflammatory bowel disease; †Patients may have met more than one criterion.

We found associations between the Day 0 pathogen (per 1.0 increase in Log10 nucleic acid/gm stool) load and mean reductions of adenovirus pathogen loads between Day 0 to 5 [0.33 Log10 nucleic acid/gm stool (95\%CI: 0.17, 0.49)]. Similarly, we found associations between Day 0 rotavirus pathogen load and the Day 0 to 5 reduction [1.05 Log10 nucleic acid/gm stool $(95 \%$ CI: $0.84,1.25)]$. However, for norovirus, no significant associations were identified. Older children experienced a greater mean norovirus pathogen load reduction [Day 5 to $28,0.08 \log 10$ nucleic acid/g stool (95\%CI: 0.02, 0.15) per 1 month increase] compared to their younger counterparts.

Although the raw baseline MVS score was associated with the rotavirus Log10 transformed stool Day 0 viral load $(P=0.05)$ in the linear regression model, the associations for norovirus $(P=$ $0.11)$ and adenovirus $(P=0.75)$ were not significant. Similarly, only the Day 5 rotavirus Log 10 transformed stool viral load was significantly associated with the follow-up MVS score $(P=0.03)$. The overall similarities of the declines in the $\log 10$ transformed pathogen loads between the probiotic and placebo groups are displayed in Figs. 3-5.

Exploratory outcomes. Sub-group analyses were conducted based on participant age and pathogen detected. In these models, the interaction between treatment assignment and age was not statistically significant when the MVS score was set as the dependent variable and the models were adjusted for other relevant covariates. Similarly, we analyzed the primary outcome, MVS score, based on breast-feeding status and found no evidence of interaction with treatment assignment; Supplementary Table 20. Adverse events did not differ between groups, as previously reported ${ }^{14}$. 


\section{Table 1 Clinical characteristics by treatment groups.}

\section{Characteristics}

Age (month), median (IQR)

Male sex (no., \%)

Weight median (IQR) (kg)

Exclusive breast fed (no., \%)

Received antibiotics in previous 14 days (no., \%)

Received rotavirus vaccine (no., \%)

Yes

No

Unsure

Duration of illness mean (SD) $(h)^{\dagger}$

Baseline modified Vesikari scale score-mean (SD)

Vomiting (no., \%)

No. of vomiting episodes in preceding $24 \mathrm{~h}$-median (IQR) $\mathrm{S}$

No. of diarrhea episodes in preceding $24 \mathrm{~h}$-median (IQR)

Febrile-no. (\%)

Clinical dehydration scale score-median (IQR)\|

Received ondansetron at index visit-no. (\%)

Received antibiotics at index visit/recommended at discharge-no. (\%)

Received intravenous rehydration at index visit-no. (\%)

Admitted to hospital at index visit-no. (\%)

\begin{tabular}{|c|c|c|}
\hline Probiotics $N=408$ & Placebo $N=408$ & $P$ - value ${ }^{t \dagger}$ \\
\hline $15.0(10.0,24.5)$ & $16.0(10.0,24.0)$ & 0.680 \\
\hline $229(56.1)$ & $235(57.6)$ & 0.724 \\
\hline $10.5(9.0,13.0)$ & $10.7(8.9,12.6)$ & 0.911 \\
\hline $22(5.4)$ & $29(7.1)$ & 0.386 \\
\hline \multirow[t]{2}{*}{$50(12.3)$} & $58(14.2)$ & 0.470 \\
\hline & & 0.827 \\
\hline $195(47.8)$ & $196(48.0)$ & \\
\hline $116(28.4)$ & $109(26.7)$ & \\
\hline $97(23.8)$ & $103(25.2)$ & \\
\hline $43.3(22.9)$ & $43.2(20.0)$ & 0.929 \\
\hline $11.2(2.7)$ & $10.9(2.8)$ & 0.161 \\
\hline $322(78.9)$ & $302(74.0)$ & 0.117 \\
\hline $4(2,6)$ & $4(2,7)$ & 0.319 \\
\hline $5(3,8)$ & $5(3,8)$ & 0.180 \\
\hline $182(44.6)$ & $179(43.9)$ & 0.888 \\
\hline $1(0,2)$ & $0(0,2)$ & 0.139 \\
\hline $90(22.1)$ & $89(21.8)$ & $>0.99$ \\
\hline $11(2.7)$ & $4(1.0)$ & 0.115 \\
\hline $36(8.8)$ & $31(7.6)$ & 0.610 \\
\hline $10(2.5)$ & $10(2.5)$ & $>0.99$ \\
\hline \multicolumn{3}{|l|}{ re enrollment, whichever } \\
\hline \multicolumn{3}{|l|}{ disease severity. } \\
\hline
\end{tabular}

Table 2 Primary outcome-modified Vesikari scale score by treatment and pathogen groups.

\begin{tabular}{|c|c|c|c|c|c|}
\hline Negative $(N=299)$ & $5.3(4.2)$ & $139 ; 5.1(4.1)$ & $160 ; 5.5(4.3)$ & $-0.410(-1.379,0.560)$ & 0.407 \\
\hline Bacteria only $(N=37)$ & $7.7(4.9)$ & $17 ; 9.4(5.3)$ & $20 ; 6.4(4.2)$ & $2.990(-0.160,6.142)$ & 0.063 \\
\hline $\begin{array}{l}\text { Virus/bacteria co-detection } \\
(N=19)\end{array}$ & $6.3(4.8)$ & $13 ; 6.2(4.4)$ & $6 ; 6.4(5.9)$ & $-0.180(-5.544,5.184)$ & 0.948 \\
\hline
\end{tabular}

\section{Discussion}

This study expands our knowledge by conducting several unique and novel virus-specific evaluations of the effectiveness of a combination probiotic in children with AGE. We found no indication that probiotic administration lessens the burden of disease, quantified by the MVS score, regardless of the etiologic pathogen group (i.e., virus, bacteria or parasite) or specific viral etiologies (i.e., adenovirus, norovirus or rotavirus). In addition, we found no evidence that children administered the probiotic agent experienced a more rapid clearance of pathogen from stool, compared to those administered placebo, either during the treatment course or over the subsequent weeks.

Earlier reports suggested that there may be pathogen-specific benefits associated with probiotic use ${ }^{29}$, with the greatest benefits seen in children with rotavirus diarrhea and limited benefit in children infected with bacterial pathogens ${ }^{30}$. Following the routine administration of a rotavirus vaccine ${ }^{31}$, norovirus has replaced rotavirus as the most common pathogen ${ }^{8}$. This pathogen shift may in part explain the lack of probiotics benefit in viral AGE in our study. While earlier studies and meta-analyses focused on evaluating the benefit of probiotics in the context of rotavirus infection, none have been sufficiently large to analyze groups of pathogens or specific viral pathogens other than rotavirus $^{32}$. In this report, we clarify that no pathogen subgroups or specific viral subgroups were found to benefit from probiotic administration. Adjustment for the duration of illness at the time of the initiation of probiotic therapy ${ }^{33}$ did not alter our findings.

Our stool pathogen load analysis constitutes a unique approach to evaluating the effect of probiotic therapy and is based on evidence that a higher stool viral load is associated with more severe disease and reflects a greater degree of intestinal epithelium damage $^{22,23,34}$. The latter scenario can lead to viral spread beyond the intestines into the bloodstream ${ }^{35,36}$. Although we hypothesized that probiotic administration may more rapidly reduce the stool pathogen load, we did not identify significant associations to support this notion. 


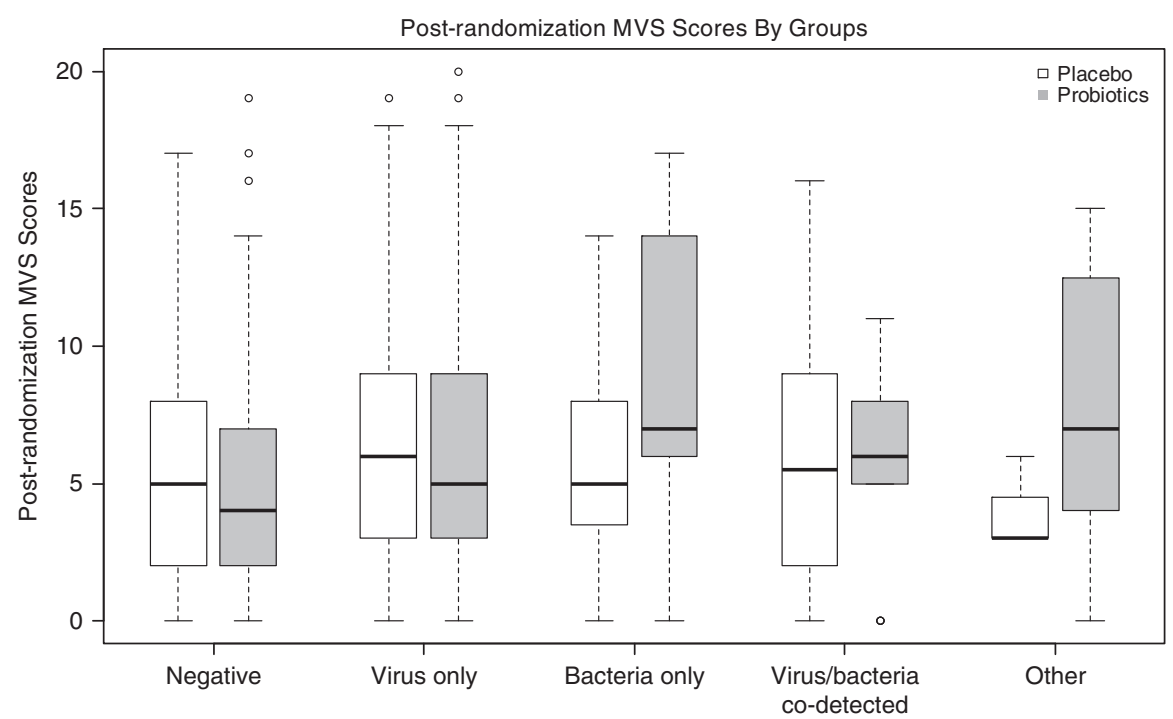

Fig. 2 Post randomization disease severity (Modified Vesikari Scale scores) based on pathogen identified, compared between probiotic and placebo groups. Boxplots of post-randomization modified Vesikari scale cores by treatment and pathogen groups (Tested negative: placebo $n=160$, probiotics $n=$ 139; virus only: placebo $n=219$, probiotics $n=232$; bacteria only: placebo $n=20$, probiotics $n=17$; virus/bacteria co-detection: placebo $n=6$; probiotics $n=13$; Other type detected including parasite only and virus/parasite co-detection: placebo $n=3$, probiotics $n=7$ ). In the box plot, the line that divides the box into two parts represents the median of the data; the upper and lower bounds of the box represent the $25 \%$ and 75 percentiles, respectively. The lower whiskers represent the $25 \%$ ile- $(1.5 \times$ IQR) and the upper whiskers represent the $75 \%$ ile- $(1.5 \times$ IQR). The dots beyond the whisker represents outliers of values within the dataset. Source data are provided as a Source Data file.

Table 3 Stool pathogen reduction, follow-up stool specimens, probiotic versus placebo, log10 transformed copies/gm stool`.

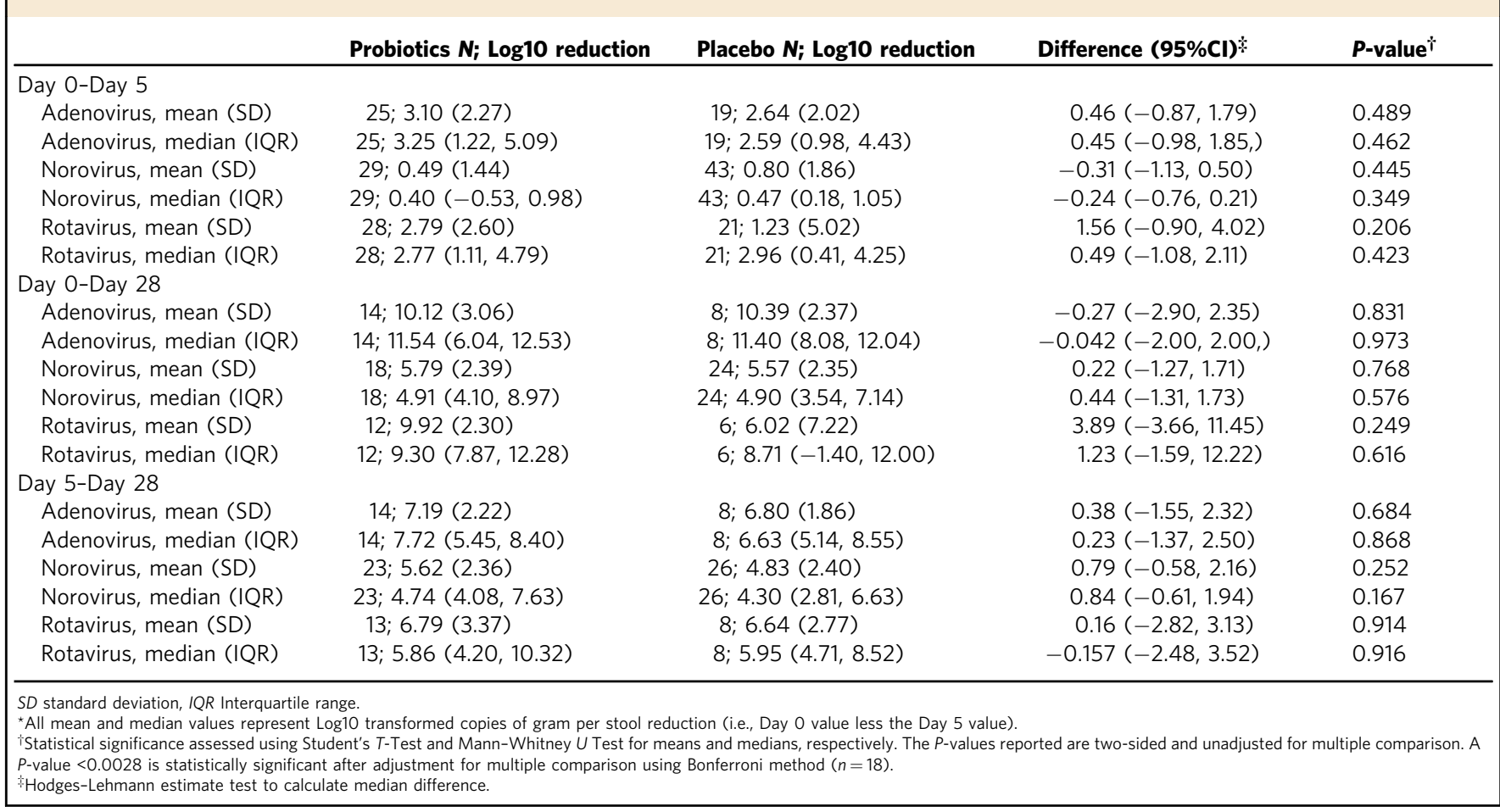

Our study has several limitations. Only $18 \%$ of participants provided specimens at all three study time points. Consequently, we had fewer participants than was anticipated and thus several secondary analyses were potentially underpowered. While participants who submitted all three specimens could have differed from those who did not, there is no reason to assume a related systematic bias. Indeed, Supplementary Table 18 demonstrates that the two groups of children were clinically similar. However, because of the small number of participants with bacterial and parasitic infections, we were unable to conduct robust pathogen load analyses related to bacteria and parasite clearance.

In conclusion, we observed no beneficial virus-specific clinical effects associated with the administration of a 5-day course of a $L$. helveticus/L. rhamnosus combination probiotic, for children with AGE. Similarly, probiotic administration did not result in more rapid clearance of viral pathogens from stool specimens, 


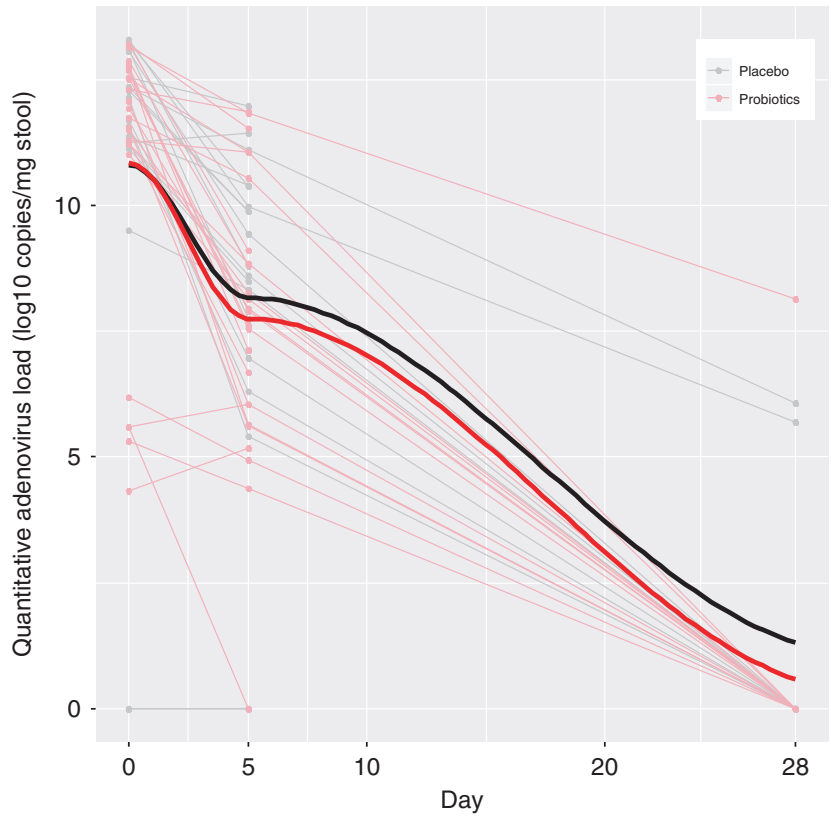

Fig. 3 Change in adenovirus viral load quantity in stool over time, compared between probiotic and placebo groups. Stool Log10 transformed copies of adenovirus by treatment group [probiotic $(N=25)$ vs. placebo $(N=20)$ ] across time (in days) following randomization on the $x$-axis. Thin light gray lines refer to patients provided placebo, this red lines refer to those provided probiotic; thick black and red lines to locally weighted smoothing lines respectively. The two-sided $P$-value represents the result of a linear mixed effect model with random intercepts (subjects random effect) comparing placebo (reference group) vs. probiotic on viral load including time and treatment group variables, and an interaction term for the latter two. Source data are provided as a Source Data file. Adenovirus (mean difference: $-0.12 ; 95 \% \mathrm{Cl}:-1.94,1.70 ; P=0.90$ ).

compared with placebo. These findings strengthen the conclusion that in children who present to an ED with viral-induced AGE, twice-daily administration of a combined L. rhamnosus/L. helveticus probiotic does not reduce the severity of AGE, or expedite the clearance of viruses in stool.

\section{Methods}

Study design and oversight. The clinical trial design and methods have been published and the trial has been registered at www.clinicaltrials.gov:

NCT01853124 $4^{37}$. The protocol was approved by the research ethics boards at each of the six participating Canadian tertiary care pediatric centers located in Calgary (Conjoint Health Research Ethics Board), London (Western University Health Sciences Research Ethics Board), Toronto (SickKids Research Ethics Board), Ottawa (Children's Hospital of Eastern Ontario Research Ethics Board), Montreal (Comité d'éthique de la recherche du CHU Sainte-Justine) and Halifax (IWK Research Ethics Board), in Canada; the caregivers of all participants provided written informed consent. In brief, in this double-blind, placebo controlled trial, patients aged 3 to 48 months with AGE presenting for ED care, were randomly assigned, in a 1:1 ratio, to receive $4.0 \times 10^{9}$ colony forming units of a L. rhamnosus R0011 and L. helveticus R0052 (95:5 ratio) probiotic preparation or matching placebo twice daily for 5 days, in addition to usual care. Eligible children had $\geq 3$ episodes of diarrhea in a 24-h period, and had vomiting or diarrhea for $<72 \mathrm{~h}$. All children were evaluated by a physician who assigned a diagnosis of AGE. Children were excluded if they or a person living in their household had a central venous line, structural heart disease, were immunocompromised, or were receiving immunosuppressive therapy. Children who presented with a history of oral or gastrointestinal surgery within the preceding 7 days, blood in their vomit or stool, bilious vomiting, a chronic intestinal disorder, pancreatic insufficiency, probiotic use in the preceding 14 days, soy allergy, and an inability to complete follow-up were also excluded.

A total of 816 participants completed 14 day follow-up and provided symptom outcome data. Attempts were made to collect stool specimens from all participants on Day 0 (ED enrollment), Day 5 (last day of probiotic/placebo administration), and Day 28 after enrollment. Only participants who tested positive for an

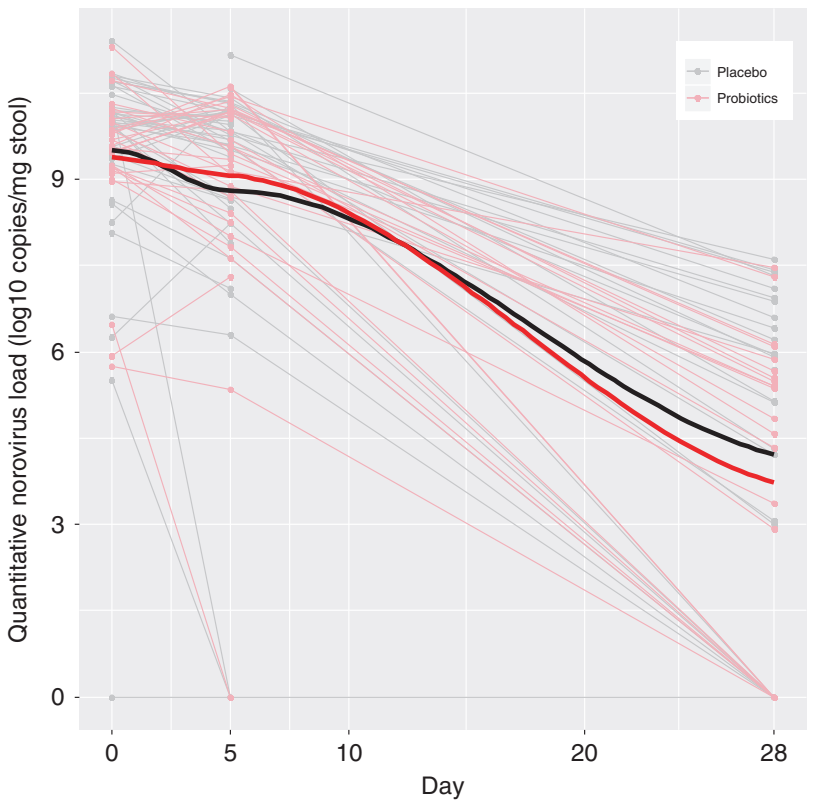

Fig. 4 Change in norovirus viral load quantity in stool over time, compared between probiotic and placebo groups. Stool Log10 transformed copies of norovirus by treatment group [probiotic $(N=34)$ vs. placebo $(N=47)$ ] across time (in days) following randomization on the $x$-axis. Thin light gray lines refer to patients provided placebo, this red lines refer to those provided probiotic; thick black and red lines to locally weighted smoothing lines respectively The two-sided $P$-value represents the result of a linear mixed effect model with random intercepts (subjects random effect) comparing placebo (reference group) vs. probiotic on viral load, including time and treatment group variables, and an interaction term for the latter two. Source data are provided as a Source Data file. Norovirus (mean difference: $0.16 ; 95 \% \mathrm{Cl}:-0.81,1.14 ; P=0.74$ ).

enteropathogen and provided stool specimens at multiple time points, were included in the current study.

Objectives. The primary objective was to determine if a 5-day probiotic treatment course administered to children with AGE resulted in pathogen-specific clinical benefits quantified using the validated and widely-used ${ }^{38}$ MVS score ${ }^{39,40}$. Secondary objectives identified a priori included (1) assessing if probiotic adminis tration resulted in a greater reduction in stool pathogen load compared with placebo; and determining the relationship between (2) correlating baseline (Day 0) stool pathogen load and baseline MVS score, and (3) Day 5 stool pathogen load and the follow-up MVS score.

Modified Vesikari scale score. MVS scores range from 0 to 20, with higher scores indicating more severe disease ${ }^{39,40}$. The MVS score quantifies severity over a broad range of symptoms and interventions among outpatients. This measurement tool was validated in two prospective cohort studies in similar patient populations ${ }^{39,40}$ and has been employed in several clinical trials ${ }^{14,15,41}$. Baseline symptoms that occurred prior to the index ED visit were not included in the follow-up MVS score calculation.

Randomization. To sequentially assign children to probiotic or placebo, we employed a random-number-generating software, accessed through www. randomize.net, which was programed to use block sizes of 4 and 6 , stratified according to site. The random allocation sequence was generated by the research pharmacy at the coordinating center. Participants were enrolled by research nurses or assistants at each site who provided caregiver with the allocation assignment. Participants and their parents or guardians, trial and clinical staff, and specimen and data analysts remained blinded to treatment assignment through the use of a placebo that was identical in appearance, smell, and weight to the intervention agent (i.e., probiotic).

Specimen collection. We attempted to collect a stool sample from all participants prior to ED discharge. If a specimen was not provided prior to ED discharge, caregivers were instructed to collect a stool sample at home, which was retrieved by a study-funded courier service. 


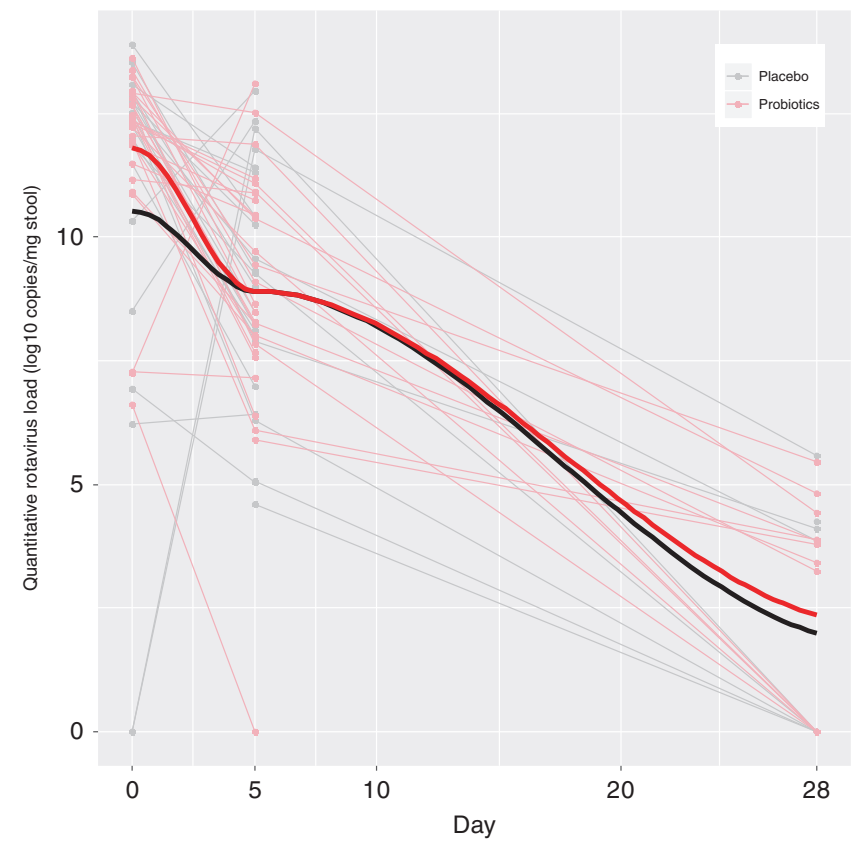

Fig. 5 Change in rotavirus viral load quantity in stool over time, compared between probiotic and placebo groups. Stool Log10

transformed copies of rotavirus by treatment group [probiotic $(N=30)$ vs. placebo $(N=24)$ ] across time (in days) following randomization on the $x$ axis. Thin light gray lines refer to patients provided placebo, this red lines refer to those provided probiotic; thick black and red lines to locally weighted smoothing lines respectively. The two-sided $P$-value represents the result of a linear mixed effect model with random intercepts (subjects random effect) comparing placebo (reference group) vs. probiotic on viral load, including time and treatment group variables, and an interaction term for the latter two. Source data are provided as a Source Data file. Rotavirus (mean difference: $0.74 ; 95 \% \mathrm{Cl}:-0.45,1.93 ; P=0.22$ ).

A rectal swab was performed with a flocked tipped sterile swab (FLOQSwabs ${ }^{\text {mp }}$ Flocked Swabs, Copan) prior to ED discharge on all children not providing a stool specimen ${ }^{19}$. This approach allowed us to obtain a specimen for molecular pathogen identification prior to probiotic administration from all study participants. The swab was only tested if an ED stool specimen was not obtained.

Day 5 and Day 28 stool samples were requested from all study participants who provided a Day 0 stool sample, either while in the ED or at home. Caregivers were provided with collection instructions along with containers. Specimens were labeled with the date and time of collection and the subject's study identification number. They were returned to the research team by a study-funded courier service within $12 \mathrm{~h}$ of collection. All specimens were placed in coolers with ice packs while in transit to the laboratory. Upon receipt, each sample was split and frozen for future testing ${ }^{42-44}$. Sites then batch-shipped all frozen stool samples to the Alberta Public Laboratories-ProvLab (Edmonton, Alberta, Canada) bi-annually to enable interim laboratory analyses to verify collection and processing procedures. All stool tests were conducted blinded to treatment allocation and clinical symptoms.

Pathogen detection. All Day 0 specimens obtained in the ED underwent bacterial culture locally. A multiplex nucleic acid panel that detects 15 pathogens: enteric viruses, bacteria and parasites (Luminex xTAG Gastrointestinal Pathogen Panel) was later performed at the Provincial Laboratory for Public Health-Alberta Public Laboratorie-ProvLab ${ }^{45}$. To ensure that negative rectal swab test results were not due to insufficient stool obtained on the rectal swab, all patients with a Day 0 ED rectal swab that tested negative for all enteropathogens had testing repeated using the Day 0 stool specimen collected at home. Day 5 and 28 specimens were tested only if the Day 0 specimen tested positive for an enteropathogen.

Pathogen load quantification. Quantification procedures were standardized to ensure that the homogeneity and proportion of stool included in each analysis was consistent between samples (intra-patient and inter-patient) and hence per reporting unit (g). To achieve this degree of standardization, a 10\% (weight/ volume) suspension of stool specimen was prepared with phosphate-buffered saline (PBS) and clarified by centrifugation. Testing of the Day 0,5 , and 28 specimens from the same patient were performed in the same test run to eliminate inter-run variation.
Viral loads were quantified as previously described ${ }^{46}$. In brief, samples were thawed, then mixed by vortexing to prepare a $10 \%$ stool specimen suspension after centrifugation. Total nucleic acid was then extracted and eluted using the MagaZorb ${ }^{\varpi}$ total RNA Prep kit (Promega, Madison, WI). Nucleic acid extracted from non-study stool samples testing positive for well-characterized enteric viruses (i.e., adenovirus 40/41, norovirus, and rotavirus) were used as positive controls. The primers and probes for the detection of adenovirus, norovirus, and rotavirus ${ }^{47-51}$ were labeled with Fam detector and Tamara quencher dyes (Applied Biosystems). Individual real-time PCR reactions for each virus were performed. After denaturing, PCR amplification was performed and profiles were collected and analyzed using Sequence Detection Software version 1.0. To quantify the three viruses, an external standard curve for each virus was established using 10 -fold dilutions from 100 copies to $1.0 \times 10^{6}$ viral $\mathrm{cDNA}$ copies/PCR ${ }^{52}$.

We employed similar methodology to quantify bacterial loads which were determined for stool samples positive for bacteria using singleplex real-time PCR assays for each respective bacteria (Campylobacter, E. coli, Salmonella, Shigella). Standard curves demonstrating the relationship between colony forming units (CFU) and crossing point of the real-time PCR assay for each organism were created by performing real-time PCR on 10 -fold dilutions of standardized bacterial suspensions that were plated onto sheep blood agar plate to determine the CFU count.

Statistical analysis. For the primary outcome we a priori anticipated that followup would be complete for $90 \%$ of clinical trial participants $(N=797)$ and all of these participants would thus have follow-up MVS scores. Based on North American ${ }^{19,53,54}$ data, we assumed the following pathogen distributions: $~ 50 \%$ viral $(N=399), \sim 40 \%$ unidentified $(N=318)$, and $\sim 10 \%$ bacterial $(N=80)$. Given our 1:1 probiotic:placebo allocation ratio, we anticipated a minimum of 40 participants per arm in the smallest group ${ }^{55}$. The proposed minimum clinically important pathogen-group and pathogen-specific MVS difference of means were based on the natural history of disease ${ }^{40}$, and the proposed benefits associated with probiotic administration (Supplementary Table 1). Power calculations assumed 40 subjects in each study arm pathogen group (i.e., probiotic and placebo virus, bacteria, and unidentified) and a standard deviation (SD) of $3.1^{40}$ around the MVS score point estimates. Based on our proposed effect sizes and assuming a minimum of 40 paired specimens for each pathogen group comparison, power was greater than $80 \%$. Similar power was present when the analysis was repeated with specific viral etiologies (smallest cell $=39$ ).

For the primary outcome, clinical benefits were evaluated by comparing the mean post-randomization MVS scores between children who received probiotics versus placebo. The difference in means was explored in relation to pathogengroup (i.e., negative, virus only, bacteria only, virus/bacteria co-detection, and other co-detection including parasite only and parasite/virus co-detection), viral (i.e., rotavirus, norovirus, adenovirus), and bacterial agents (i.e., Campylobacter and Salmonella spp.). For co-detections (i.e., multiple enteropathogens detected), we first included all cases with the specific pathogen, then repeated the analysis using only single pathogen detection cases. Children under two years of age from whom only Clostridioides difficile was detected, were counted as test-negative as young children are often colonized with this agent ${ }^{53,56}$. To assess for main effects, the analysis employed a weighted linear regression model that included treatment, pathogen, interaction terms for treatment assignment and pathogen groups along with other key covariates (i.e., age, sex, MVS score at enrollment, pre-index visit or index visit hospitalization, and pre-index visit antibiotic use).

Stool pathogen load quantification values were $\log 10$ transformed in keeping with standards approaches to reporting nucleic acid concentrations in human body fluids. The secondary outcome of stool pathogen load reduction was quantified as the difference in the number of copies of pathogen-specific NA/gm between Days 0 and 5 (i.e., Day $0-$ Day $5=$ stool pathogen load reduction) and Day 5 and 28. Multivariable regression models including treatment, pathogen and other key covariates (e.g., day of illness, age, sex, MVS score at enrollment, hospitalization, antibiotic use, baseline pathogen load) were constructed. Locally weighted smoothing lines were constructed and compared between groups.

Correlations between (1) the baseline (Day 0) stool pathogen load and the baseline MVS score and (2) the Day 5 stool pathogen load with the follow-up MVS score, were performed employing within pathogen-group and within pathogen (virus) linear regression analyses. All regression models were adjusted for a priori identified variables as described for the primary outcome.

All reported regression models employed variable transformations when model residuals were non-normally distributed and the transformations, if performed, are reported. Models were only constructed to evaluate pathogens identified in a minimum of 10 participants. Multiple imputation was used to account for individual missing elements of the 7-item MVS score. Time, but not date, of the first or last vomit or diarrheal episodes, were the most commonly missing variable, absent in a maximum of $24 \%$ of participants. The imputation model, based on inspection, assumed that data were missing at random and included key baseline characteristics, trial group, and all efficacy outcomes ${ }^{14}$. All statistical tests were two-sided; overall statistical tests of significance for the primary outcomes was set at 0.005 using the Bonferroni approach to correct for the 10 comparisons performed.

Reporting summary. Further information on research design is available in the Nature Research Reporting Summary linked to this article. 


\section{Data availability}

The full study protocol and the datasets, which includes all data fields reported in this study, are available, following manuscript publication, upon request from the corresponding author (Dr. Stephen Freedman, Stephen.Freedman@AlbertaHealthServices.ca), following the provision of ethics approval. The source data underlying Figs. 2 and $3 \mathrm{a}-\mathrm{c}$ are provided as a Source Data file.

Received: 18 December 2019; Accepted: 25 April 2020;

Published online: 21 May 2020

\section{References}

1. Thomas, M. K., Murray, R., Nesbitt, A. \& Pollari, F. The incidence of acute gastrointestinal illness in Canada, foodbook survey 2014-2015. Can. J. Infect. Dis. Med. Microbiol. 2017, 5956148, https://doi.org/10.1155/2017/5956148 (2017).

2. Scallan, E. et al. Foodborne illness acquired in the United States-major pathogens. Emerg. Infect. Dis. 17, 7-15 (2011).

3. Scallan, E., Griffin, P. M., Angulo, F. J., Tauxe, R. V. \& Hoekstra, R. M. Foodborne illness acquired in the United States-unspecified agents. Emerg. Infect. Dis. 17, 16-22 (2011).

4. Aliabadi, N. et al. Global impact of rotavirus vaccine introduction on rotavirus hospitalisations among children under 5 years of age, 2008-16: findings from the Global Rotavirus Surveillance Network. Lancet Glob. Health 7, e893-e903 (2019).

5. Shah, M. P., Dahl, R. M., Parashar, U. D. \& Lopman, B. A. Annual changes in rotavirus hospitalization rates before and after rotavirus vaccine implementation in the United States. PLoS ONE 13, e0191429, https://doi.org/ 10.1371/journal.pone.0191429 (2018).

6. Payne, D. C. et al. Effectiveness of pentavalent and monovalent rotavirus vaccines in concurrent use among US children $<5$ years of age, 2009-2011. Clin. Infect. Dis. 57, 13-20 (2013).

7. Collaborators, G. B. D. D. D. Estimates of the global, regional, and national morbidity, mortality, and aetiologies of diarrhoea in 195 countries: a systematic analysis for the Global Burden of Disease Study 2016. Lancet Infect. Dis. 18, 1211-1228 (2018).

8. Payne, D. C. et al. Norovirus and medically attended gastroenteritis in U.S. Child. N. Engl. J. Med. 368, 1121-1130 (2013).

9. Vanderkooi, O. G. et al. A prospective comparative study of children with gastroenteritis: emergency department compared with symptomatic care at home. Eur. J. Clin. Microbiol. Infect. Dis. 38, 2371-2379 (2019).

10. Allen, S. J., Martinez, E. G., Gregorio, G. V. \& Dans, L. F. Probiotics for treating acute infectious diarrhoea. Cochrane Database Syst. Rev. CD003048, https://doi.org/10.1002/14651858.CD003048.pub3 (2010).

11. Lo Vecchio, A. et al. Comparison of recommendations in clinical practice guidelines for acute gastroenteritis in children. J. Pediatr. Gastroenterol. Nutr. 63, 226-235 (2016).

12. Guarino, A. et al. European Society for Pediatric Gastroenterology, Hepatology, and Nutrition/European Society for Pediatric Infectious Diseases evidence-based guidelines for the management of acute gastroenteritis in children in Europe: update 2014. J. Pediatr. Gastroenterol. Nutr. 59, 132-152 (2014).

13. Farthing, M. et al. Acute diarrhea in adults and children: a global perspective. J. Clin. Gastroenterol. 47, 12-20 (2013).

14. Freedman, S. B. et al. Multicenter trial of a combination probiotic for children with gastroenteritis. N. Engl. J. Med. 379, 2015-2026 (2018).

15. Schnadower, D. et al. Lactobacillus rhamnosus GG versus placebo for acute gastroenteritis. Child. N. Engl. J. Med. 379, 2002-2014 (2018).

16. Szymanski, $\mathrm{H}$. et al. Treatment of acute infectious diarrhoea in infants and children with a mixture of three Lactobacillus rhamnosus strains-a randomized, double-blind, placebo-controlled trial. Aliment. Pharmacol. Ther. 23, 247-253 (2006)

17. Navaneethan, U. \& Giannella, R. A. Mechanisms of infectious diarrhea. Nat. Clin. Pract. Gastroenterol. Hepatol. 5, 637-647 (2008).

18. Corr, S. C., Hill, C. \& Gahan, C. G. Understanding the mechanisms by which probiotics inhibit gastrointestinal pathogens. Adv. Food Nutr. Res. 56, 1-15 (2009).

19. Freedman, S. B. et al. Enteropathogen detection in children with diarrhoea, or vomiting, or both, comparing rectal flocked swabs with stool specimens: an outpatient cohort study. Lancet Gastroenterol. Hepatol. 2, 662-669 (2017).

20. Hall, A. J. et al. Norovirus disease in the United States. Emerg. Infect. Dis. 19, 1198-1205 (2013)

21. Phillips, G. et al. Diagnosing norovirus-associated infectious intestinal disease using viral load. BMC Infect. Dis. 9, 63, https://doi.org/10.1186/1471-2334-963 (2009).

22. He, T. et al. Norovirus loads in stool specimens of cancer patients with norovirus gastroenteritis. J. Mol. diagnostics: JMD 19, 836-842 (2017).
23. Reymao, T. K. A. et al. Norovirus RNA in serum associated with increased fecal viral load in children: detection, quantification and molecular analysis. PLoS ONE 13, e0199763, https://doi.org/10.1371/journal.pone.0199763 (2018).

24. Fumian, T. M. et al. Quantitative and molecular analysis of noroviruses RNA in blood from children hospitalized for acute gastroenteritis in Belem, Brazil. J. Clin. Virol. 58, 31-35 (2013).

25. Huhti, L., Hemming-Harlo, M. \& Vesikari, T. Norovirus detection from sera of young children with acute norovirus gastroenteritis. J. Clin. Virol. 79, 6-9 (2016).

26. Furuya, D. et al. Age, viral copy number, and immunosuppressive therapy affect the duration of norovirus RNA excretion in inpatients diagnosed with norovirus infection. Jpn J. Infect. Dis. 64, 104-108 (2011).

27. Cybulski, R. J. Jr. et al. Clinical impact of a multiplex gastrointestinal polymerase chain reaction panel in patients with acute gastroenteritis. Clin. Infect. Dis. 67, 1688-1696 (2018).

28. Abbasi, J. Are probiotics money down the toilet? or worse? JAMA 321, 633-635 (2019).

29. Ahmadi, E., Alizadeh-Navaei, R. \& Rezai, M. S. Efficacy of probiotic use in acute rotavirus diarrhea in children: a systematic review and meta-analysis. Casp. J. Intern Med 6, 187-195 (2015).

30. Szajewska, H. et al. Systematic review with meta-analysis: lactobacillus rhamnosus GG for treating acute gastroenteritis in children - a 2019 update. Aliment. Pharmacol. Ther. 49, 1376-1384 (2019).

31. Committee on Infectious. D. \& American Academy of, P. Prevention of rotavirus disease: updated guidelines for use of rotavirus vaccine. Pediatrics 123, 1412-1420 (2009).

32. Erdogan, O. et al. The comparition of the efficacy of two different probiotics in rotavirus gastroenteritis in children. J. Trop. Med 2012, 787240, https://doi. org/10.1155/2012/787240 (2012)

33. Brussow, H. Probiotics and prebiotics in clinical tests: an update. F1000Res $\mathbf{8}$ https://doi.org/10.12688/f1000research.19043.1 (2019).

34. Justino, M. C. A. et al. Rotavirus antigenemia as a common event among children hospitalised for severe, acute gastroenteritis in Belem, northern Brazil. BMC Pediatr. 19, 193, https://doi.org/10.1186/s12887-019-1535-2 (2019).

35. Sugata, K. et al. Analysis of rotavirus antigenemia and extraintestinal manifestations in children with rotavirus gastroenteritis. Pediatrics 122, 392-397 (2008).

36. Ramani, S. et al. Rotavirus antigenemia in Indian children with rotavirus gastroenteritis and asymptomatic infections. Clin. Infect. Dis. 51, 1284-1289 (2010).

37. Freedman, S. B. et al. Impact of emergency department probiotic treatment of pediatric gastroenteritis: study protocol for the PROGUT (Probiotic Regimen for Outpatient Gastroenteritis Utility of Treatment) randomized controlled trial. Trials 15, 170, https://doi.org/10.1186/1745-6215-15-170 (2014).

38. Riera-Montes, M., O'Ryan, M. \& Verstraeten, T. Norovirus and rotavirus disease severity in children: systematic review and meta-analysis. Pediatr. Infect. Dis. J. 37, 501-505 (2018).

39. Freedman, S. B., Eltorky, M. \& Gorelick, M. \& Pediatric Emergency Research Canada Gastroenteritis Study, G. Evaluation of a gastroenteritis severity score for use in outpatient settings. Pediatrics 125, e1278-e1285 (2010).

40. Schnadower, D. et al. Validation of the modified Vesikari score in children with gastroenteritis in 5 US emergency departments. J. Pediatr. Gastroenterol. Nutr. 57, 514-519 (2013)

41. Bernstein, D. I. et al. Norovirus vaccine against experimental human GII.4 virus illness: a challenge study in healthy adults. J. Infect. Dis. 211, 870-878 (2015).

42. Denno, D. M. et al. Etiology of diarrhea in pediatric outpatient settings. Pediatr. Infect. Dis. J. 24, 142-148 (2005).

43. Denno, D. M. et al. Diarrhea etiology in a pediatric emergency department: a case control study. Clin. Infect. Dis. 55, 897-904 (2012).

44. Jones, T. F. et al. Use of stool collection kits delivered to patients can improve confirmation of etiology in foodborne disease outbreaks. Clin. Infect. Dis. 39, 1454-1459 (2004)

45. Perry, M. D., Corden, S. A. \& Howe, R. A. Evaluation of the Luminex xTAG Gastrointestinal Pathogen Panel and the Savyon Diagnostics Gastrointestinal Infection Panel for the detection of enteric pathogens in clinical samples. J. Med. Microbiol. 63, 1419-1426 (2014).

46. Pang, X. L., Preiksaitis, J. K. \& Lee, B. Multiplex real time RT-PCR for the detection and quantitation of norovirus genogroups I and II in patients with acute gastroenteritis. J. Clin. Virol. 33, 168-171 (2005).

47. Kageyama, T. et al. Broadly reactive and highly sensitive assay for Norwalklike viruses based on real-time quantitative reverse transcription-PCR. J. Clin Microbiol. 41, 1548-1557 (2003).

48. Pang, X. L. et al. Increased detection of rotavirus using a real time reverse transcription-polymerase chain reaction (RT-PCR) assay in stool specimens from children with diarrhea. J. Med. Virol. 72, 496-501 (2004). 
49. Oka, T. et al. Detection of human sapovirus by real-time reverse transcriptionpolymerase chain reaction. J. Med. Virol. 78, 1347-1353 (2006).

50. Pang, X. L. et al. Pre-analytical and analytical procedures for the detection of enteric viruses and enterovirus in water samples. J. Virol. Methods 184, 77-83 (2012).

51. Iturriza-Gomara, M., Kang, G. \& Gray, J. Rotavirus genotyping: keeping up with an evolving population of human rotaviruses. J. Clin. Virol. 31, 259-265 (2004).

52. Qiu, Y. et al. UV inactivation of human infectious viruses at two full-scale wastewater treatment plants in Canada. Water Res .147, 73-81 (2018).

53. Nicholson, M. R. et al. Using multiplex molecular testing to determine the etiology of acute gastroenteritis in children. J. Pediatr. 176, 50-56 e52 (2016).

54. Stockmann, C. et al. Detection of 23 gastrointestinal pathogens among children who present with diarrhea. J. Pediatr. Infect. Dis. Soc. 6, 231-238 (2017).

55. Vesikari, T. et al. Efficacy of two doses of RIT 4237 bovine rotavirus vaccine for prevention of rotavirus diarrhoea. Acta Paediatr. Scand. 80, 173-180 (1991).

56. Kim, A. et al. Epidemiology and factors related to clinical severity of acute gastroenteritis in hospitalized children after the introduction of rotavirus vaccination. J. Korean Med. Sci. 32, 465-474 (2017).

\section{Acknowledgements}

We thank the participants and their families for trusting us to conduct this trial; the trial investigators and support staff across all sites for their commitment to the successful and diligent conduct of the trial; the site coordinators, including Marie-Christine Auclair, R.N., B.Sc.N., Zach Cantor, B.A., Kamary Coriolano Da Silva, Ph.D., Dale Dalgleish, B.H Sc.N., Eleanor Fitzpatrick, M.N., Kelly Kim, B.Sc., B.A., Maggie Rumantir, M.D., Judy Sweeney, R.N., B.Sc.N., and Avery Yandt, B.Sc., B.A., for trial coordination; the staff of Lallemand Health Solutions, Calgary Laboratory Services, Alberta Health Services, Research Pharmacy, Provincial Laboratory for Public Health, Edmonton and Calgary, Alberta, for supporting the conduct of this trial; the emergency department physicians, nurses, and ancillary staff at all trial sites; the Pediatric Emergency Medicine Research Associates' Program at Alberta Children's Hospital, the Students Undertaking a Pediatric Program of Research Training program at the Children's Hospital of Eastern Ontario, and the staff of the Pediatric Research Academic Initiative in SickKids Emergency program at the Hospital for Sick Children, for identifying potentially eligible trial participants; and Dr. Marie Louie (Calgary) for building the connections that made the microbiologic investigations possible. No compensation for the assistance provided by any aforementioned persons was provided. This study was supported by the Canadian Institutes of Health Research (grants 286384 and 325412), the Alberta Children's Hospital Foundation Professorship in Child Health and Wellness (to Dr. Freedman), a grant from the Alberta Children's Hospital Foundation to the Pediatric Emergency Medicine Research Associates' Program, Calgary Laboratory Services (in kind), Provincial Laboratory for Public Health-Alberta Public Laboratories, Luminex, and Copan Italia. P.M.S. is the recipient of a Canada Research Chair in Gastrointestinal Disease.

\section{Author contributions}

S.F. and Y.F. conceived the study, obtained funding, and S.F. wrote the first draft. S.F., X.P., L.C., B.L., P.S., D.S., and Y.F. designed the study with help from the other authors.
S.W., S.S., S.G. K.F., N.P., and K.H. were responsible for clinical operations. S.W. oversaw all study activities. X.P., L.C., B.L. were in charge of developing microbiology protocols and standard operating procedures. Y.Q., B.G., C.L. was in charge of conducting all laboratory analyses and managed microbiology quality assurance. J.X. and A.N. conducted the data management and statistical analyses. S.W. ensured adherence to the protocol and handled data acquisition and institutional review board matters. All authors contributed in writing different sections of the manuscripts.

\section{Competing interests}

None of the authors have any potential or perceived conflicts of interest to declare. The study sponsors played no role in study design, conduct, data acquisition, analysis, manuscript preparation or the decision to submit the manuscript for publication. Dr. Stephen Freedman provides consulting services to Takeda Pharmaceuticals Inc. and was an invited speaker at the 10th Probiotics, Prebiotics and New Foods, Nutraceuticals and Botanicals for Nutrition and Human and Microbiota Health. PMS serves on advisory boards for Antibe Therapeutics, Cargill and Nestle-Gerber. He has received honoraria from Abbott Nutrition for speaking at continuing medical education activities and received research support from Lallemand Human Nutrition for a postdoctoral fellow award through a Mitacs Accelerates Internship.

\section{Additional information}

Supplementary information is available for this paper at https://doi.org/10.1038/s41467020-16308-3.

Correspondence and requests for materials should be addressed to S.B.F.

Peer review information Nature Communications thanks the anonymous reviewers for their contribution to the peer review of this work. Peer reviewer reports are available.

Reprints and permission information is available at http://www.nature.com/reprints Publisher's note Springer Nature remains neutral with regard to jurisdictional claims in published maps and institutional affiliations.

\begin{abstract}
(c) (i)
Open Access This article is licensed under a Creative Commons Attribution 4.0 International License, which permits use, sharing, adaptation, distribution and reproduction in any medium or format, as long as you give appropriate credit to the original author(s) and the source, provide a link to the Creative Commons license, and indicate if changes were made. The images or other third party material in this article are included in the article's Creative Commons license, unles indicated otherwise in a credit line to the material. If material is not included in the article's Creative Commons license and your intended use is not permitted by statutory regulation or exceeds the permitted use, you will need to obtain permission directly from the copyright holder. To view a copy of this license, visit http://creativecommons.org/ licenses/by/4.0/.
\end{abstract}

(c) The Author(s) 2020

\section{the Pediatric Emergency Research Canada Probiotic (PERC) Regimen for Outpatient Gastroenteritis Utility of Treatment (PROGUT) Trial Group}

Stephen B. Freedman', Jianling Xie ${ }^{1}$, Alberto Nettel-Aguirre', Xiao-Li Pang ${ }^{2}$, Linda Chui ${ }^{2}$ Sarah Williamson-Urquhart', David Schnadower ${ }^{3}$, Suzanne Schuh ${ }^{4}$, Philip M. Sherman ${ }^{4}$, Bonita Lee ${ }^{2}$, Serge Gouin ${ }^{5}$, Ken J. Farion' ${ }^{6}$, Naveen Poonai ${ }^{7}$, Katrina F. Hurley ${ }^{8}$, Yuanyuan Qiu², Binal Ghandi², Colin Lloyd², Yaron Finkelstein ${ }^{4}$, Andrew R. Willan ${ }^{4}$, Ron Goeree ${ }^{9}$, David W. Johnson ${ }^{1}$, Karen Black ${ }^{10}$, Marc H. Gorelick ${ }^{11}$

${ }^{9}$ PATH Research Institute, St. Joseph's Healthcare Hamilton, 25 Main Street West, Suite 2000, Hamilton, ON L8P 1H1, Canada. ${ }^{10}$ University of British Columbia, 4480 Oak St, Vancouver, BC V6H 3N1, Canada. ${ }^{11}$ University of Minnesota, 2525 Chicago Ave, Minneapolis, MN 55404, USA. 